\title{
Preoperative chemotherapy with docetaxel, cisplatin, and 5-fluorouracil for locally advanced esophageal carcinosarcoma: a case report and review of the literature
}

Tomoko Yoshimoto ${ }^{1}$, Shinichiro Kobayashi ${ }^{1}$, Kengo Kanetaka ${ }^{1}$, Kazuma Kobayashi', Yasuhiro Nagata ${ }^{2}$, Michi Morita', Yuriko Isagawa', Naoe Kinoshita ${ }^{3}$, Mitsuhisa Takatsuki ${ }^{1}$ and Susumu Eguchi ${ }^{{ }^{*}}$

\begin{abstract}
Background: Esophageal carcinosarcoma is a relatively rare malignant neoplasm composed of both epithelial carcinomatous and mesenchymal sarcomatous elements. There is no recommended clinical treatment for esophageal carcinosarcoma because of the rarity of the disease. This report describes a case of esophageal carcinosarcoma that was effectively treated with docetaxel, cisplatin, and 5-fluorouracil as preoperative chemotherapy.

Case presentation: A 73-year-old man had a chief complaint of dysphagia with epigastric pain. Esophagogastroduodenoscopy (EGD) revealed a polypoid neoplasm combined with an infiltrative ulcer that exhibited a mixture of squamous cell carcinoma and spindle cell sarcoma histologically. Computed tomography findings showed swollen lymph nodes in the mediastinum and around the cardia. We diagnosed esophageal carcinosarcoma CT3N1M0 cStage III. After preoperative chemotherapy with docetaxel, cisplatin, and 5-fluorouracil, the patient underwent thoracoscopic esophagectomy with three-field lymph node dissection. Histological findings revealed that the sarcomatous component had completely disappeared and the carcinomatous component was only confined by the basement membrane with scar formation of the muscularis propria. Mural fibrotic lesions were observed in several resected regional lymph nodes. Hence, immediately after preoperative therapy, the esophageal carcinosarcoma was diagnosed as ypTisNOMO fStage I. The patient remained alive without tumor recurrence at 12 months after the operation.

Conclusions: A review of the literature revealed that there is still no established therapeutic strategy for locally advanced esophageal carcinosarcoma, especially against the sarcomatous component. We herein provide the first report in which the sarcomatous component showed a complete response to preoperative chemotherapy with docetaxel, cisplatin, and 5-fluorouracil. Preoperative chemotherapy with docetaxel, cisplatin, and 5-fluorouracil followed by esophagectomy with extended lymphadenectomy may achieve definitive treatment for locally advanced esophageal carcinosarcoma.
\end{abstract}

Keywords: Docetaxel, Cisplatin, 5-Fluorouracil, Esophagus, Carcinosarcoma

\footnotetext{
* Correspondence: sueguchi@nagasaki-u.ac.jp

'Department of Surgery, Nagasaki University Graduate School of Biomedical

Sciences, Sakamoto 1-7-1, Nagasaki 852-8102, Japan

Full list of author information is available at the end of the article
} 


\section{Background}

Esophageal carcinosarcoma is a relatively rare malignant neoplasm composed of both epithelial carcinomatous elements and mesenchymal sarcomatous elements [1, 2]. Although esophageal carcinosarcoma has a different cellular pleomorphism, the sarcomatous elements are considered to have a monoclonal epithelial origin with sarcomatoid differentiation. Because of the rarity of the disease and lack of evidence regarding the response of the sarcomatous element, no robust conclusion has been reached about whether perioperative therapy can improve the control of locally advanced esophageal carcinosarcoma. We herein report a case of locally advanced esophageal carcinosarcoma treated with docetaxel, cisplatin, and 5-fluorouracil (DCF) as preoperative chemotherapy.

\section{Case presentation}

A 73-year-old man was admitted to a clinic with epigastric pain. His family history was unremarkable, and he took medication for hypertension. When he was referred to our hospital for further examination, his epigastric pain had improved and his physical findings were normal. All laboratory data and serum tumor markers were within the normal limits. Esophagogastroduodenoscopy (EGD) revealed a large polypoid neoplasm combined with an infiltrative ulcer, $6 \mathrm{~cm}$ in diameter, in the thoracic esophagus (Fig. 1a). The histological findings of a biopsy specimen from the tumor showed squamous cell carcinoma with spindle cell components. Immunohistochemical evaluation of the biopsy specimen revealed that the squamous cell carcinoma cells were positive for pancytokeratin and p63, while the spindle cells were positive

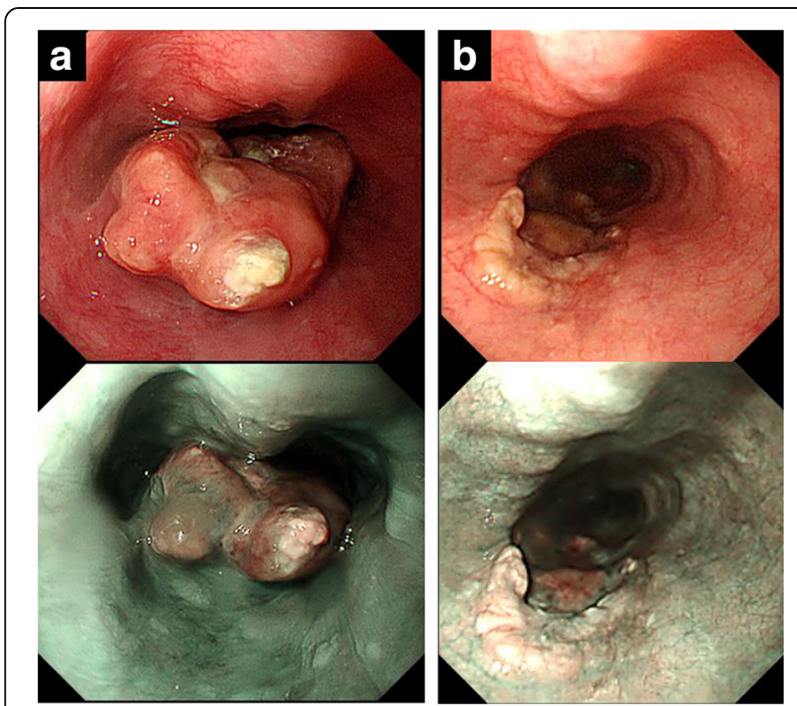

Fig. 1 Endoscopic findings. a A protruding tumor was observed in the middle of the thoracic esophagus. $\mathbf{b}$ After two courses of preoperative chemotherapy, the tumor had reduced in size for vimentin (Fig. 2). Transitional features between the carcinomatous and sarcomatous components were evident (Fig. 3). Therefore, the tumor was diagnosed as carcinosarcoma. A computed tomography (CT) examination revealed that the neoplasm expanded into the thoracic esophagus without invasion to the adjacent organs and that swollen lymph nodes were present in the mediastinum and around the cardia (Fig. 4a). No metastatic regions were found in distant organs, including the lungs and liver. Fluorodeoxyglucose-positron emission tomography (FDG-PET) showed high FDG uptake by the neoplasm in the esophagus and swollen lymph nodes in the mediastinum without involvement of distant lymph nodes and organs (Fig. 5a). The clinical diagnosis according to the eighth edition of the Union for International Cancer Control (UICC) was T3N1M0 Stage III esophageal cancer. Preoperative DCF chemotherapy was proposed. The DCF therapy, which consisted of intravenous docetaxel $\left(60 \mathrm{mg} / \mathrm{m}^{2}\right.$, day 1$)$, cisplatin $\left(60 \mathrm{mg} / \mathrm{m}^{2}\right.$, day 1$)$, and continuous 5fluorouracil $\left(800 \mathrm{mg} / \mathrm{m}^{2}\right.$, days $\left.1-5\right)$, was administered twice at an interval of 4 weeks [3]. After two courses of DCF therapy, the neoplasm was extremely reduced on EGD (Fig. 1b), and FDG-PET and CT examinations showed no FDG uptake by the tumor and lymph nodes (Figs. 4b and 5b). Severe neutropenia and stomatitis developed during the second course of DCF therapy. The patient underwent thoracoscopic esophagectomy with three-field lymph node dissection after the preoperative chemotherapy. The resected specimen revealed a scar with no obvious neoplasm (Fig. 6a). The histological findings revealed a nodular scar, $4 \mathrm{~cm}$ in diameter, within the muscularis propria that seemed to be a degenerated tumor. The sarcomatous element had completely disappeared, and the carcinomatous element was only observed in situ (Fig. 6b). Several scars were observed in the resected regional lymph nodes. Hence, immediately after preoperative therapy, the esophageal carcinosarcoma was diagnosed as ypTisNOMO Stage I according to the eighth edition of the UICC. He was discharged on postoperative day 17 with no complications. He remained disease-free for 12 months after the surgery.

\section{Discussion}

Esophageal carcinosarcoma is a rare malignant neoplasm that accounts for 0.1 to $2.8 \%$ of all esophageal malignant tumors [1]. In general, esophageal carcinosarcoma tends to present as a bulky intraluminal polypoid mass and to occur more commonly in men, typically those aged 60 to 70 years. About $60 \%$ of tumors arise in the midesophagus, with nearly one third in the distal esophagus [4]. The prognosis of esophageal carcinosarcoma remains unclear because of the rarity of the disease. Esophageal carcinosarcoma may have a better prognosis than 
a

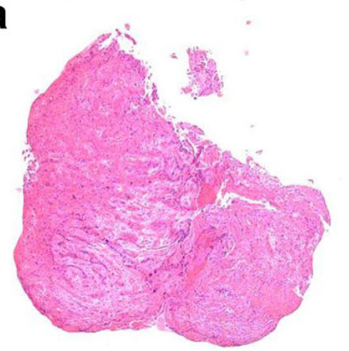

b

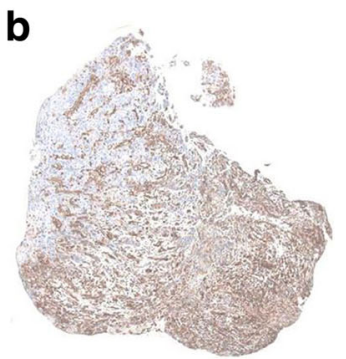

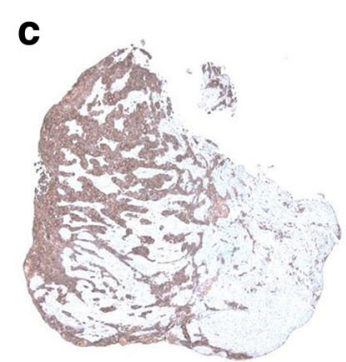

Fig. 2 Microscopic and immunohistochemical findings of a biopsy specimen from the tumor. a The histological findings of the biopsy specimen showed squamous cell carcinoma with spindle cell components. b, c Immunohistochemical evaluation of the biopsy specimen revealed that the squamous cell carcinoma cells were positive for pan-cytokeratin, while the spindle cells were positive for vimentin. $\mathbf{a}$ Hematoxylin and eosin, $\mathbf{b}$ vimentin, and c pan-cytokeratin $(\times 40)$

esophageal squamous cell carcinoma because the early onset of symptoms caused by intraluminal growth allows earlier detection of the disease $[1,2,4]$. Meanwhile, some investigators have reported a high frequency of distant metastasis and poorer long-term prognosis [5-7]. Kuo et al. [7] reported that the 1- and 2-year survival rates in 12 patients with esophageal carcinosarcoma were 50 and $25 \%$, respectively. Other systematic reviews have shown that patients with T1 esophageal carcinosarcoma had a higher rate of lymph node metastasis and a lower 5-year survival rate than patients with T1 esophageal squamous cell carcinoma [5, 6]. Iyomasa et al. [8] reported that patients with esophageal carcinosarcoma sometimes developed late recurrence in the form of hematogenous metastasis after surgery. Thus, aggressive treatments are required for not only the carcinomatous component but also the sarcomatous component in locally advanced esophageal carcinosarcoma.

Esophageal carcinosarcoma is defined as a neoplasm with both epithelial carcinomatous and sarcomatous components [2]. Immunohistochemical analysis is necessary for the diagnosis of carcinosarcoma. Immunohistochemically, epithelial membrane antigen, carcinoembryonic antigen, cytokeratin, and synaptophysin are highly specific markers for carcinomatous elements, while vimentin, smooth muscle antigen, and desmin are specific for sarcomatous elements $[6,9]$. Although the pathogenesis of the sarcomatous component in esophageal carcinosarcoma remains unclear, the sarcomatous component may arise from epithelial-mesenchymal transition (EMT) of the carcinomatous component $[10,11]$. In carcinosarcoma, tumor cells are expected to lose their epithelial phenotype and

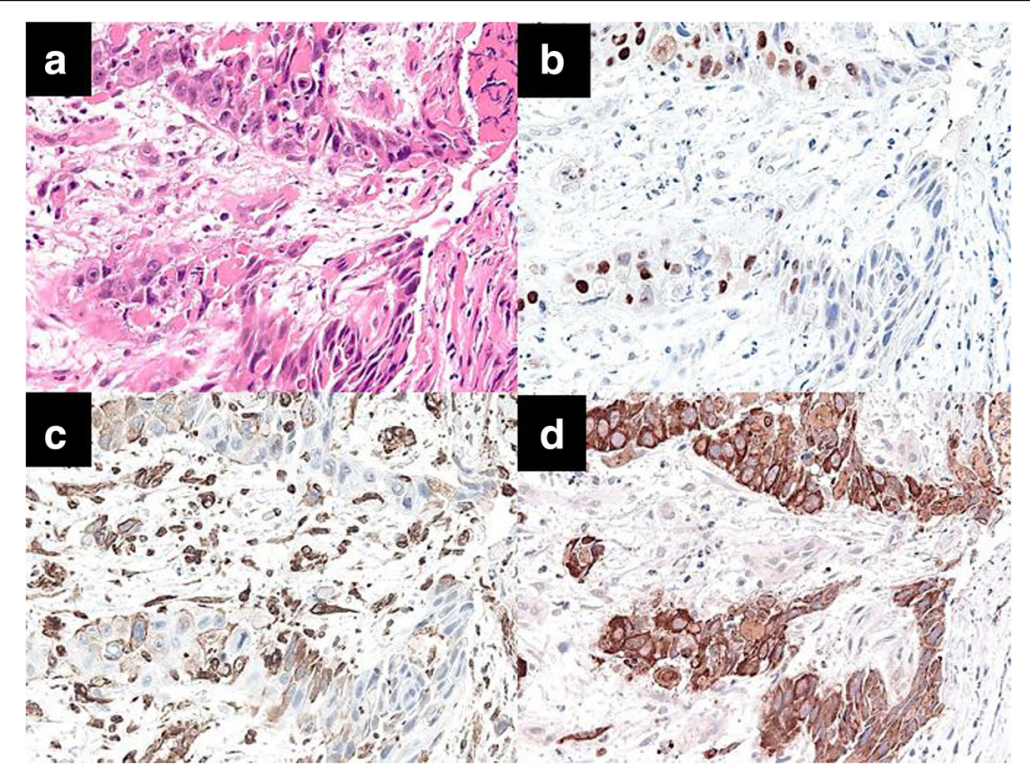

Fig. 3 Microscopic and immunohistochemical findings of a biopsy specimen from the transitional zone between the carcinomatous and sarcomatous components. a-d $\mathbf{~ I n}$ the transitional zone between the two components, there were scattered malignant cells expressing pan-cytokeratin, vimentin, or p63. a Hematoxylin and eosin, b p63, c vimentin, and d pan-cytokeratin $(\times 200)$ 

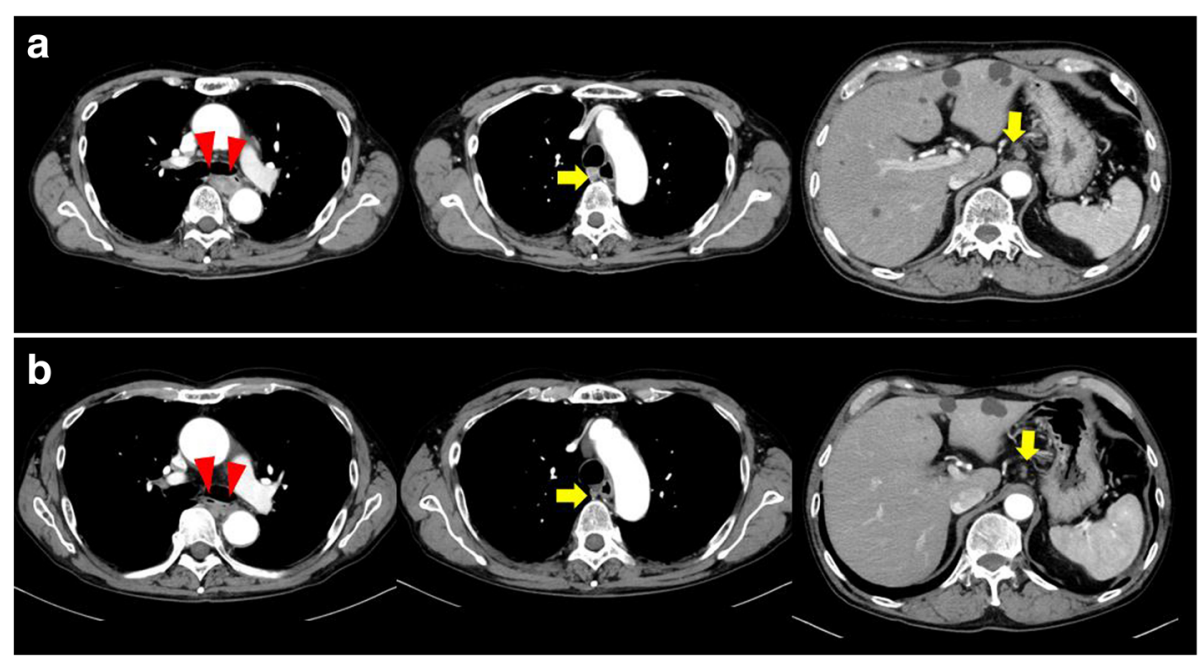

Fig. 4 Computed tomography findings. a Before preoperative chemotherapy, a tumor was observed in the middle of the esophagus (red arrows) with swollen lymph nodes in the mediastinum and around the cardia (yellow arrows), but there was no distant metastasis. b After preoperative chemotherapy, the tumor (red arrows) and swollen lymph nodes in the mediastinum and around the cardia (yellow arrows) were reduced

gradually and sequentially acquire a mesenchymal phenotype during the EMT process [10]. In the present case, the biopsy specimen from the tumor was composed of both carcinomatous and sarcomatous elements. The carcinomatous component was positive for cytokeratin, while the sarcomatous component was positive for vimentin. A transitional zone was observed between the two components. The transitional zone consisted of scattered carcinoma cells expressing vimentin or p63, a marker of progenitor cells associated with inhibition of EMT-positive cells in the transitional zone [12]. In the present case, all malignant cells in the carcinomatous element were positive for p63, while almost all malignant cells in the sarcomatous element were negative for $\mathrm{p} 63$. The biological features of the transitional zone suggested that the sarcomatous element may have originated from the carcinomatous element via EMT.

There is no recommended clinical treatment for esophageal carcinosarcoma because of the rarity of the disease. Esophageal carcinomas have been treated with the multidisciplinary approach of surgery, chemotherapy, and radiotherapy. In Japan, preoperative chemotherapy with cisplatin and 5-fluorouracil followed by esophagectomy and regional lymph node resection is the standard treatment for locally advanced esophageal squamous cell carcinoma [13]. In addition, preoperative DCF chemotherapy has been reported to be safe and tolerable in patients with locally advanced esophageal cancer [3].

Docetaxel is an anticancer agent used to treat sarcomas in several fields [14]. The efficacy of docetaxel for
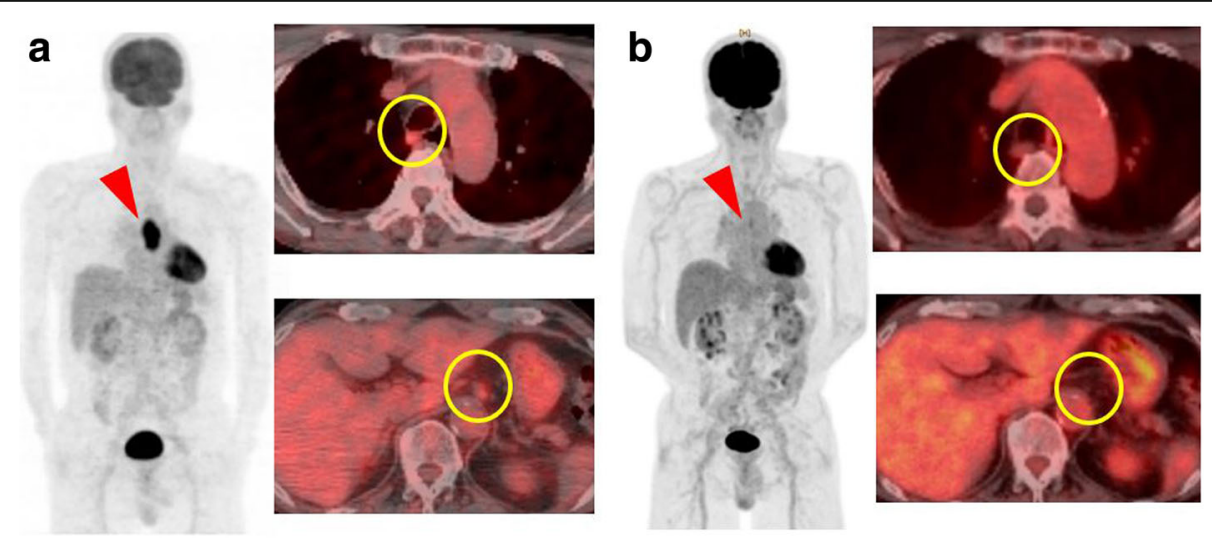

Fig. 5 Fluorodeoxyglucose-positron emission tomography (FDG-PET) findings. a Before preoperative chemotherapy, there was high FDG uptake by the tumor in the esophagus (a red arrow) and the swollen lymph nodes in the mediastinum and around the cardia (yellow circles). $\mathbf{b}$ After preoperative chemotherapy, the tumor had almost disappeared (a red arrow) and the swollen lymph nodes in the mediastinum and around the cardia had decreased (yellow circles) 


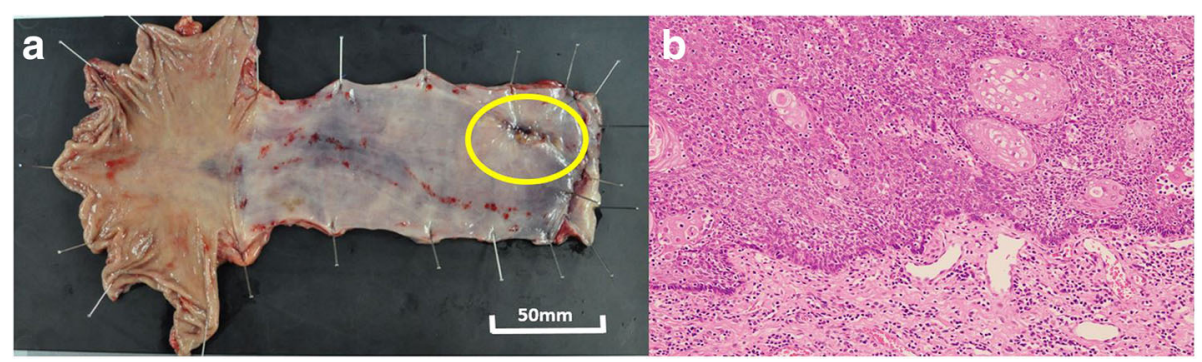

Fig. 6 Macroscopic and microscopic findings of the resected specimen. a The scar without an obvious neoplasm measured $40 \mathrm{~mm}$ (bar $50 \mathrm{~mm}$ ). b A histopathological examination demonstrated that only the carcinomatous component was present in situ and the sarcomatous component had disappeared $(\times 200)$

bone and soft tissue sarcomas has been proven, mainly in combination regimens with gemcitabine [15-17]. Investigators in the gynecological field reported that leiomyosarcoma and endometrial stromal sarcoma treated with docetaxel-based chemotherapy showed favorable response rates [18-20]. Thus, we selected DCF therapy as preoperative therapy in the present case. In fact, the endoscopic findings revealed that the tumor dramatically decreased in size, although the clinical therapeutic effect of DCF therapy was stable disease according to the Response Evaluation Criteria in Solid Tumors guideline [21]. The postoperative pathological examination showed the carcinomatous element was slightly retained, but no sarcomatous element was observed. Therefore, the patient was classified as having a near pathological complete response, defined as 1 to $10 \%$ residual tumor cells with no tumor remaining in the resected lymph nodes [22]. DCF therapy would be effective for the carcinomatous element, while docetaxel may also be effective for the sarcomatous element.

We searched for articles in PubMed published since 1990 containing the key terms "esophagus" and "carcinosarcoma." We identified seven cases of esophageal carcinosarcoma treated with preoperative therapy [7, 23-25]. Table 1 shows the details of these cases as well as our case. Six patients underwent chemoradiotherapy, and only one patient was treated with chemotherapy. A clinical response to preoperative therapy was observed in six patients (three patients with a partial response and three patients with stable disease). A pathological response to preoperative therapy was observed in four patients, including three patients treated with chemoradiotherapy or DCF therapy who obtained a near pathological complete response. Meanwhile, one patient treated with 5-fluorouracil and cisplatin obtained an insufficient pathological response to preoperative therapy. Thus, chemoradiotherapy or DCF therapy may be accepted as preoperative therapy for downstaging of locally advanced esophageal carcinosarcoma.

FDG-PET scans are well established for determining the stage, prognosis, and efficacy of preoperative therapy in squamous cell carcinoma [26]. Moreover, the standardized uptake values of carcinosarcoma are higher than those of squamous cell carcinoma regardless of differentiation type because the sarcomatous element of carcinosarcoma shows higher FDG uptake than the carcinomatous element $[27,28]$. In the present case, the sarcomatous element completely disappeared after preoperative chemotherapy in the pathological evaluation as shown by FDG-PET scans, which showed no FDG uptake by the tumor and lymph nodes. Thus, FDG-PET scans can be a useful tool for predicting the therapeutic response and surveillance of esophageal carcinosarcoma.

Table 1 Esophageal carcinosarcoma treated with preoperative therapy

\begin{tabular}{lllllllll}
\hline First author & Year & Age & Sex & Stage & Preoperative therapy & Clinical response & Pathological response & Prognosis \\
\hline Zuiki [23] & 2009 & 50 & Male & II & CRT (FP+62 Gy) & PR & ND & months alive \\
Zuiki [23] & 2009 & 66 & Male & I & CRT (FP+40.2 Gy) & PR & ND & 19 months alive \\
Kobayashi [24] & 2010 & 68 & Male & III & CRT (S-1/CDDP+40 Gy) & PR & npCR & 60 months alive \\
Kobayashi [24] & 2010 & 64 & Male & III & CRT (FP+38 Gy) & SD & npCR & 11 months dead \\
Kuo [7] & 2010 & 68 & Male & III & CRT (ND) & ND & ND & 27 months alive \\
Kuo [7] & 2010 & 45 & Male & IV & CRT (ND) & ND & NC & 6 months alive \\
Kobayashi [25] & 2015 & 69 & Male & II & FP & SD & npCR & 60 months alive \\
Present case & 2016 & 73 & Male & III & DCF & SD & 12 months alive \\
\hline
\end{tabular}

$C R T$ chemoradiotherapy, $P R$ partial response, $n P C R$ near pathological complete response, SD stable disease, FP 5-fluorouracil and cisplatin, S-1/CDDP S-1 and cisplatin, NC no change, DCF docetaxel, cisplatin, and 5-fluorouracil, ND not described 


\section{Conclusions}

We have described a case of esophageal carcinosarcoma that was effectively treated with preoperative DCF chemotherapy. Preoperative chemotherapy with DCF may be an option for locally advanced esophageal carcinosarcoma.

\section{Abbreviations}

CT: Computed tomography; DCF: Docetaxel, cisplatin, and 5-fluorouracil; EGD: Esophagogastroduodenoscopy; EMT: Epithelial-mesenchymal transition; FDG-PET: Fluorodeoxyglucose-positron emission tomography; UICC: Union for International Cancer Control

\section{Acknowledgements}

The authors thank Alison Sherwin, PhD, and Angela Morben, DVM, ELS, from Edanz Group (www.edanzediting.com/ac) for editing a draft of this manuscript.

\section{Funding}

This study was supported by a Grant-in-Aid for Scientific Research from the Japan Society for the Promotion of Science (17K16569 to Shinichiro Kobayashi).

\section{Authors' contributions}

All of the authors have read and approved the manuscript. TY and SK collected, analyzed, and interpreted the patient disease data and edited the manuscript. TY and SK contributed equally. KKa and KKo supervised the patient treatment and research project. MM and $\mathrm{YI}$ are clinitians who participated in the treatments of the patient and discussions. YN and MT participated in discussions. NK is a pathologist who participated in the diagnosis of esophageal carcinosarcoma. SE approved the final submission of the manuscript.

\section{Ethics approval and consent to participate}

The publication of the present study was undertaken in accordance with the ethical standards of our institution (17061947).

\section{Consent for publication}

Informed consent was obtained from the patient and his family for the publication of this report.

\section{Competing interests}

The authors declare that they have no competing interests.

\section{Publisher's Note}

Springer Nature remains neutral with regard to jurisdictional claims in published maps and institutional affiliations.

\section{Author details}

${ }^{1}$ Department of Surgery, Nagasaki University Graduate School of Biomedical Sciences, Sakamoto 1-7-1, Nagasaki 852-8102, Japan. ${ }^{2}$ Center for Comprehensive Community Care Education, Nagasaki University Graduate School of Biomedical Sciences, Sakamoto 1-12-4, Nagasaki 852-8523, Japan. ${ }^{3}$ Department of Pathology, Saiseikai Nagasaki Hospital, Katafuchi 2-5-1, Nagasaki 850-0003, Japan.

Received: 21 November 2017 Accepted: 5 February 2018 Published online: 17 February 2018

\section{References}

1. Wang $L$, Lin $Y$, Long $H$, Liu H, Rao H, He $Y$, et al. Esophageal carcinosarcoma: a unique entity with better prognosis. Ann Surg Oncol. 2013;20:997-1004.

2. Japan Esophageal Society. Japanese classification of esophageal cancer, 11th edition: part II and III. Esophagus. 2017;14:37-65.

3. Hara H, Tahara M, Daiko H, Kato K, Igaki H, Kadowaki S, et al. Phase II feasibility study of preoperative chemotherapy with docetaxel, cisplatin, and fluorouracil for esophageal squamous cell carcinoma. Cancer Sci. 2013;104:1455-60.

4. Raza MA, Mazzara PF. Sarcomatoid carcinoma of esophagus. Arch Pathol Lab Med. 2011;135:945-8.

5. Sanada Y, Hihara J, Yoshida K, Yamaguchi Y. Esophageal carcinosarcoma with intramural metastasis. Dis Esophagus. 2006;19:119-31.
6. Sano A, Sakurai S, Kato H, Sakai M, Tanaka N, Inose T, et al. Clinicopathological and immunohistochemical characteristics of esophageal carcinosarcoma. Anticancer Res. 2009;29:3375-80.

7. Kuo CJ, Lin TN, Lin CJ, Wu RC, Chang HK, Chu YY, et al. Clinical manifestation of esophageal carcinosarcoma: a Taiwan experience. Dis Esophagus. 2010:23:122-7.

8. Iyomasa S, Kato H, Tachimori Y, Watanabe H, Yamaguchi H, Itabashi M. Carcinosarcoma of the esophagus: a twenty-case study. Jpn J Clin Oncol. 1990;20:99-106

9. Xu F, Zou WB, Li XP, Xu YM, Qi XF, Hu LH, et al. Multiple carcinosarcomas of the esophagus and stomach. Oncol Lett. 2013;5:1017-21.

10. Sung CO, Park CK, Kim SH. Classification of epithelial-mesenchymal transition phenotypes in esophageal squamous cell carcinoma is strongly associated with patient prognosis. Mod Pathol. 2011;24:1060-8.

11. Sung CO, Choi H, Lee KW, Kim SH. Sarcomatoid carcinoma represents a complete phenotype with various pathways of epithelial mesenchymal transition. J Clin Pathol. 2013:66:601-6.

12. Zhang Y, Yan W, Chen X. P63 regulates tubular formation via epithelial-tomesenchymal transition. Oncogene. 2014:33:1548-57.

13. Ando $N$, Kato $H$, Igaki $H$, Shinoda M, Ozawa S, Shimizu H, et al. A randomized trial comparing postoperative adjuvant chemotherapy with cisplatin and 5-fluorouracil versus preoperative chemotherapy for localized advanced squamous cell carcinoma of the thoracic esophagus (JCOG9907). Ann Surg Oncol. 2012;19:68-74.

14. Ravi V, Patel S, Benjamin RS. Chemotherapy for soft-tissue sarcomas. Oncology (Williston Park). 2015;29:43-50.

15. Pautier P, Floquet A, Penel N, Piperno-Neumann S, Isambert N, Rey A, et al. Randomized multicenter and stratified phase II study of gemcitabine alone versus gemcitabine and docetaxel in patients with metastatic or relapsed leiomyosarcomas: a Federation Nationale des Centres de Lutte Contre le Cancer (FNCLCC) French Sarcoma Group study (TAXOGEM study). Oncologist. 2012:17:1213-20.

16. Takahashi M, Komine K, Imai H, Okada Y, Saijo K, Shirota H, et al. Efficacy and safety of gemcitabine plus docetaxel in Japanese patients with unresectable or recurrent bone and soft tissue sarcoma: results from a single-institutional analysis. PLoS One. 2017;12:e0176972.

17. Choi Y, Yun MS, Lim SH, Lee J, Ahn JH, Kim YJ, et al. Gemcitabine and docetaxel combination for advanced soft tissue sarcoma: a nationwide retrospective study. Cancer Res Treat. 2017. https://doi.org/10.4143/crt.2016.535.

18. Hensley ML, Blessing JA, Mannel R, Rose PG. Fixed-dose rate gemcitabine plus docetaxel as first-line therapy for metastatic uterine leiomyosarcoma: a Gynecologic Oncology Group phase II trial. Gynecol Oncol. 2008;109:329-34.

19. Hensley ML, Blessing JA, Degeest K, Abulafia O, Rose PG, Homesley HD. Fixed-dose rate gemcitabine plus docetaxel as second-line therapy for metastatic uterine leiomyosarcoma: a Gynecologic Oncology Group phase II study. Gynecol Oncol. 2008;109:323-8.

20. Maeda O, Moritani S, Ichihara S, Inoue T, Ishihara Y, Yamamoto S, et al. Long-term survival in low-grade endometrial stromal sarcoma with childbirth and multidisciplinary treatment: a case report. J Med Case Rep. 2015;9:233

21. Therasse P, Arbuck SG, Eisenhauer EA, Wanders J, Kaplan RS, Rubinstein L, et al. New guidelines to evaluate the response to treatment in solid tumors. J Natl Cancer Inst. 2000;92:205-16.

22. Shapiro J, ten Kate FJ, van Hagen P, Biermann K, Wijnhoven BP, van Lanschot JJ. Residual esophageal cancer after neoadjuvant chemoradiotherapy frequently involves the mucosa and submucosa. Ann Surg. 2013;258:678-88. discussion 88-9

23. Zuiki T, Hosoya Y, Ui T, Haruta H, Kurashina K, Saito S, et al. Therapeutic effectiveness of chemoradiotherapy for carcinosarcoma of the esophagus: two case reports and a review of the literature. Esophagus. 2009;6:189-95.

24. Kobayashi D, Koike M, Kodera Y, Fujiwara M, Nakayama G, Nakao A. Carcinosarcoma of the esophagus treated with chemoradiotherapy: report of four cases. Esophagus. 2010;7:119-25.

25. Kobayashi S, Nagata Y, Tokai H, Ito M, Fujioka H. Multidisciplinary therapy for granulocyte-colony-stimulating factor producing carcinosarcoma of the esophagus: report of a case. Clin Case Rep. 2015; 3:681-5.

26. Fletcher JW, Djulbegovic B, Soares HP, Siegel BA, Lowe VJ, Lyman GH, et al. Recommendations on the use of 18F-FDG PET in oncology. J Nucl Med. 2008;49:480-508. 
27. Fukunaga T, Okazumi S, Koide $Y$, Isono K, Imazeki K. Evaluation of esophageal cancers using fluorine-18-fluorodeoxyglucose PET. J Nucl Med. 1998;39:1002-7.

28. Matsutani T, Nomura T, Hagiwara N, Yoshida H, Yokoyama T, Katayama H, et al. A case of carcinosarcoma of the esophagus detected on fluorodeoxyglucose positron emission tomography. J Nippon Med Sch. 2014;81:401-5.

\section{Submit your manuscript to a SpringerOpen ${ }^{\circ}$} journal and benefit from:

- Convenient online submission

- Rigorous peer review

- Open access: articles freely available online

- High visibility within the field

- Retaining the copyright to your article

Submit your next manuscript at $\gg$ springeropen.com 